\title{
Mixed-Potential Integral Equation Based Characteristic Mode Analysis of Microstrip Antennas
}

\author{
Yikai Chen, Liwen Guo, and Shiwen Yang \\ University of Electronic Science and Technology of China, Chengdu 611731, China \\ Correspondence should be addressed to Yikai Chen; ykchen@uestc.edu.cn
}

Received 26 August 2016; Accepted 1 November 2016

Academic Editor: Miguel Ferrando Bataller

Copyright ( 2016 Yikai Chen et al. This is an open access article distributed under the Creative Commons Attribution License, which permits unrestricted use, distribution, and reproduction in any medium, provided the original work is properly cited.

\begin{abstract}
A characteristic mode (CM) formulation is developed for the modal analysis of microstrip antennas. It is derived from the mixedpotential integral equation (MPIE) with spatial-domain Green's functions for multilayered media, where spatial-domain Green's functions take into account the effect of the multilayered media. The resultant characteristic currents and fields are orthogonal with each other among different orders of modes. Together with the eigenvalues and their deduced indicators, the CMs provide deep physical insights into the radiation mechanisms of microstrip antennas. Numerical results are presented to confirm CM formulation's effectiveness and accuracy in determining the resonant frequencies, radiating mode currents, and modal fields of microstrip antennas. As opposed to the very popular CM formulation for conducting bodies, comparative studies are presented to show the quite different modal analysis results by considering the multilayered media.
\end{abstract}

\section{Introduction}

Microstrip antennas (MSAs) have gained great interest in modern wireless communications due to their attractive features such as low profile, light weight, and ease of fabrication. Nevertheless, MSAs usually have narrow bandwidths and can only operate effectively in the vicinity of resonant frequencies. Therefore, it is important to accurately determine the resonant frequencies of MSAs. Moreover, obtaining the radiating modes of MSAs is also of great importance for practical designs. A number of methods have been proposed to determine resonant frequencies of MSAs. These methods can be generally classified into analytical and numerical methods. Analytical methods such as cavity models $[1,2]$ and transmission-line [3] provide intuitive physical explanations to the radiation mechanism. However, they are only suitable for some canonic structures. Corrected formulae for irregular shaped MSAs become quite complicated [4]. Although electromagnetic modeling techniques like the method of moments (MoM) are well developed for MSA analysis, feedings have to be properly designed to get the true radiating modes. In [5], the MoM was employed to determine the resonances of irregularly shaped MSAs by seeking the frequencies at which the real part of the surface current density is maximum and the imaginary part is zero. Although this method was effective in finding resonant frequencies, the modal fields (or modal currents) strongly depend on the incident plane wave used in the MoM simulation.

Recently, the characteristic mode (CM) theory [6] for conducting bodies, which is based on the electric field integral equation (EFIE), has been employed for modal analysis of MSAs. In [7, 8], resonant frequency of the fundamental $\mathrm{TM}_{10}$ mode for rectangular patch antennas with air substrate is analyzed using the CM theory for conducting bodies. Similarly, U-slot and E-shaped MSAs with air substrate were also analyzed using the CM theory for conducting bodies [9]. However, dielectric material with $\varepsilon_{r}>1$ is used extensively in MSA designs. As will be demonstrated, the CM theory for conducting bodies has many limitations in the modal analysis of MSAs.

This paper develops a CM formulation from the mixedpotential integral equation (MPIE). Spatial-domain Green's functions (GFs) of multilayered media are used in the MPIE to account for the effect of multilayered media. Numerical 
results are given to show the accuracy of the proposed CM formulation. Comparative studies are also given to show the limitations of the CM theory of conducting bodies in the modal analysis of MSAs with nonair dielectric substrate.

\section{CM Theory for MPIE with Multilayered Media GF}

The conventional CM theory was initially developed by Garbacz and Turpin [10] and then refined by Harrington and Mautz [6] in 1971. The resultant CMs define a set of orthogonal currents that can be used to expand the induced current due to external sources. Because of such attractive properties, the CM theory has been extensively applied in various antenna designs [11-13]. In order to adapt the CM theory to MSAs analysis, CM formulation for MSAs with multilayered media is developed.

The MPIE with spatial-domain GFs of multilayered media (infinite in transverse directions) allows accurate modeling surface currents in multilayered media environment $[14,15]$. We assume that a microstrip antenna is illuminated by an incident wave $\mathbf{E}^{i}$ and the total tangential electric field must vanish on the conducting patches. It reduces to the following MPIE:

$$
\left[j \omega \mu_{0}\left\langle G_{a}, \mathbf{J}\right\rangle-\frac{1}{j \omega \varepsilon_{0}} \nabla\left\langle G_{q}, \nabla^{\prime} \cdot \mathbf{J}\right\rangle\right]_{\tan }=\mathbf{E}_{\tan }^{i},
$$

where $\mathbf{J}$ is the unknown surface current and $G_{a}$ and $G_{q}$ are the spatial-domain GFs for dyadic magnetic vector and electric scalar potential for multilayered media, respectively. The spatial-domain GFs can be computed from an inverse Hankel transform of their spectral-domain counterparts $\widetilde{G}_{a, q}$,

$$
\begin{aligned}
& G_{a, q}\left(\mathbf{r}, \mathbf{r}^{\prime}\right) \\
& \quad=\frac{1}{4 \pi} \int_{-\infty}^{+\infty} d k_{\rho} \rho \widetilde{G}_{a, q}\left(k_{\rho} ; z, z^{\prime}\right) H_{0}^{(2)}\left(k_{\rho}\left|\boldsymbol{\rho}-\boldsymbol{\rho}^{\prime}\right|\right) .
\end{aligned}
$$

The spatial-domain GFs are able to account for the effects of the grounded multilayered media in MSAs. Hence, the CM formulation developed from MPIE can better capture the resonant frequencies and radiating mode currents of MSAs. Due to the computational expense for the numerical integration of the highly oscillatory integrand, several efficient algorithms have been developed to accelerate the numerical integration. To date, the discrete complex image method (DCIM) [14, 15] has mostly been used for radiating patches buried in multilayered media. The DCIM is based on extracting the quasi-static part and surface wave contributions from the spectral-domain GFs and then approximating the remainder as a series of complex exponential functions. The DCIM depends on Sommerfeld's identity:

$$
\frac{e^{-j k r}}{r}=-\frac{j}{2} \int_{-\infty}^{+\infty} d k_{\rho} \rho H_{0}^{(2)}\left(k_{\rho}\left|\boldsymbol{\rho}-\boldsymbol{\rho}^{\prime}\right|\right) \frac{e^{-j k_{z}|z|}}{k_{z}} .
$$

Therefore, if the spectral-domain GF is approximated in terms of exponential functions, the inverse Hankel transformation in (2) can be evaluated analytically in a closed form for each exponential term. The CPU time for the numerical evaluation of the transformation in (2) is thus reduced. Hence, the DCIM is implemented in this paper for the spatialdomain GFs evaluations.

After the spatial-domain GFs is calculated, the MPIE can be easily discretized using the MoM procedure. By applying the standard Galerkin's procedure to the MPIE with using the RWG basis functions $\mathbf{f}_{m}(\mathbf{r})$, the elements of the resultant impedance matrix $\mathbf{Z}^{\mathrm{MPIE}}$ can be computed from the following:

$$
\begin{aligned}
& \mathbf{Z}_{m n}^{\mathrm{MPIE}}=j \omega \mu_{0} \int_{T_{m}} \int_{T_{n}} \mathbf{f}_{m}(\mathbf{r}) \cdot \mathbf{f}_{n}\left(\mathbf{r}^{\prime}\right) G_{a}\left(\mathbf{r}, \mathbf{r}^{\prime}\right) d \mathbf{r}^{\prime} d \mathbf{r} \\
& \quad+\frac{1}{j \omega \varepsilon_{0}} \\
& \quad \cdot \int_{T_{m}} \int_{T_{n}} \nabla \cdot \mathbf{f}_{m}(\mathbf{r}) \nabla^{\prime} \cdot \mathbf{f}_{n}\left(\mathbf{r}^{\prime}\right) G_{q}\left(\mathbf{r}, \mathbf{r}^{\prime}\right) d \mathbf{r}^{\prime} d \mathbf{r} .
\end{aligned}
$$

As observed from (3), the impedance matrix $\mathbf{Z}^{\mathrm{MPIE}}$ is symmetric. Following the derivations in [6], an MPIE based generalized eigenvalue equation can be obtained for CM analysis:

$$
\mathbf{X}^{\mathrm{MPIE}} \mathbf{J}_{n}=\lambda_{n} \mathbf{R}^{\mathrm{MPIE}} \mathbf{J}_{n}
$$

where $\mathbf{R}^{\mathrm{MPIE}}$ and $\mathbf{X}^{\mathrm{MPIE}}$ are the real and imaginary parts of $\mathrm{Z}^{\mathrm{MPIE}}$. Both of them are symmetric matrix. Moreover, $\mathbf{R}^{\mathrm{MPIE}}$ is also a semidefinite matrix. $\lambda_{n}$ is the eigenvalue associated with each characteristic current $\mathbf{J}_{n}$. Because of the symmetry of $\mathbf{R}^{\mathrm{MPIE}}$ and $\mathbf{X}^{\mathrm{MPIE}}$, both of $\mathbf{J}_{n}$ and $\lambda_{n}$ are real.

The characteristic current is generally normalized according to $\left\langle\mathbf{J}_{n}, \mathbf{R}^{\mathrm{MPIE}} \cdot \mathbf{J}_{n}\right\rangle=1$, such that each characteristic current $\mathbf{J}_{n}$ radiates unit radiation power. After the normalization, the characteristic currents satisfy the following orthogonal relationships:

$$
\begin{aligned}
& \left\langle\mathbf{J}_{m}, \mathbf{R}^{\mathrm{MPIE}} \cdot \mathbf{J}_{n}\right\rangle=\left\langle\mathbf{J}_{m}^{*}, \mathbf{R}^{\mathrm{MPIE}} \cdot \mathbf{J}_{n}\right\rangle=\delta_{m n} \\
& \left\langle\mathbf{J}_{m}, \mathbf{X}^{\mathrm{MPIE}} \cdot \mathbf{J}_{n}\right\rangle=\left\langle\mathbf{J}_{m}^{*}, \mathbf{X}^{\mathrm{MPIE}} \cdot \mathbf{J}_{n}\right\rangle=\lambda_{n} \delta_{m n},
\end{aligned}
$$

where $\delta_{m n}$ is the Kronecker delta function.

The electric field $\mathbf{E}_{n}$ and magnetic field $\mathbf{H}_{n}$ radiated by the characteristic current $\mathbf{J}_{n}$ are termed as characteristic fields. Making use of the complex Poynting theorem, orthogonal relationships for the characteristic fields are obtained [6],

$$
\begin{aligned}
P\left(\mathbf{J}_{m}, \mathbf{J}_{n}\right)= & \left\langle\mathbf{J}_{m}^{*}, \mathbf{Z}^{\mathrm{MPIE}} \cdot \mathbf{J}_{n}\right\rangle \\
= & \left\langle\mathbf{J}_{m}^{*}, \mathbf{R}^{\mathrm{MPIE}} \cdot \mathbf{J}_{n}\right\rangle+j\left\langle\mathbf{J}_{m}^{*}, \mathbf{X}^{\mathrm{MPIE}} \cdot \mathbf{J}_{n}\right\rangle \\
= & \left(1+j \lambda_{n}\right) \delta_{m n} \\
= & \oiint_{S^{\prime}}\left(\mathbf{E}_{m}^{*} \times \mathbf{H}_{n}\right) \cdot d \mathbf{s} \\
& +j \omega \iiint_{V}\left(\mu \mathbf{H}_{m}^{*} \cdot \mathbf{H}_{n}-\varepsilon \mathbf{E}_{m}^{*} \cdot \mathbf{E}_{n}\right) d v .
\end{aligned}
$$


In practical radiation problems, the radiating field of a microstrip antenna is of finite extent. If we choose the radiation surface $S^{\prime}$ in (7) to be the radiation sphere at infinite far-field region, we have

$$
\frac{1}{\eta} \oiint_{S_{\infty}} \mathbf{E}_{m}^{*} \cdot \mathbf{E}_{n} d s=\delta_{m n} .
$$

Equation (8) illustrates that the characteristic fields form an orthogonal set in the far-field region. The orthogonality of characteristic currents and fields can be also observed from the numerical results in Section 3.

Let $m=n$ in (7); we can see that the complex power due to the characteristic current $\mathbf{J}_{m}$ consists of two parts: the radiated power (real power) and stored energy (imaginary power):

$$
\begin{aligned}
P_{\text {real }} & =\oiint_{S_{\infty}}\left(\mathbf{E}_{m}^{*} \times \mathbf{H}_{m}\right) \cdot d \mathbf{s}=1 \\
P_{\text {imag }} & =j \omega \iiint_{V}\left(\mu \mathbf{H}_{m}^{*} \cdot \mathbf{H}_{m}-\varepsilon \mathbf{E}_{m}^{*} \cdot \mathbf{E}_{m}\right) d v=j \lambda_{m} .
\end{aligned}
$$

Apparently, characteristic mode with $\lambda_{m}=0$ does not contribute to imaginary power. It indicates that such radiating mode can be fully excited for radiation purpose. However, for those inefficient characteristic modes with larger $\left|\lambda_{m}\right|$, current density with larger amplitude is required to give unit radiating power, because large amount of energy is stored within the system. Therefore, for modes with the same current density, the radiated power (i.e., real power) for mode $\lambda_{m}=0$ is maximized as compared with those that have larger $\left|\lambda_{m}\right|$. This is the most direct way to understand how the magnitude of an eigenvalue is related to the behavior of a mode. Generally, eigenvalue $\lambda_{m}$ ranges from $-\infty$ to $+\infty$. Equation (10) clearly shows that the sign of the eigenvalue indicates whether a mode stores more electric energy than magnetic energy $\left(\lambda_{m}<0\right)$ or vice versa $\left(\lambda_{m}>0\right) . \lambda_{m}=0$ indicates that the structure is in modal resonance.

Other than the eigenvalues tell how the mode can be efficiently excited for radiation purpose, modal significance is also a popular indicator in understanding resonant behaviors:

$$
\mathrm{MS}_{n}=\frac{1}{\left|1+j \lambda_{n}\right|} .
$$

The modal significance represents the contribution of a particular mode to the total radiation responses when an ideal external source is applied to fully excite the mode. In particular, $\mathrm{MS}_{n}=1$ indicates that the structure is in resonance at the calculated frequency. In addition, characteristic angle is also of great importance for antenna designs where multiple modes need to be excited for certain radiation performance (e.g., circular polarization) [9]. It is defined as follows:

$$
\alpha_{n}=180^{\circ}-\tan ^{-1} \lambda_{n}
$$

It denotes the phase delay angle between the characteristic current and its associated characteristic field on the radiating surface.

\section{Numerical Results and Discussion}

To confirm the validity of the MPIE based CM theory, MSAs with single layered and three-layered dielectric substrates are investigated. Comparative study is performed to intuitively show the differences between the CM theory of conducting bodies and the MPIE based CM theory for multilayered media.

3.1. Rectangular Patch Antenna. A rectangular MSA with dimensions of $L \times W=76 \times 50 \mathrm{~mm}^{2}$ was analyzed from $0.8 \mathrm{GHz}$ to $2.2 \mathrm{GHz}$. The patch is printed on a grounded dielectric substrate with relative permittivity $\varepsilon_{r}=3.38$ and thickness $h=1.524 \mathrm{~mm}$. In [2], the resonant frequencies of the first four modes are computed using the cavity model. The modes $\mathrm{TM}_{10}, \mathrm{TM}_{01}, \mathrm{TM}_{11}$, and $\mathrm{TM}_{20}$ resonate at $1.075 \mathrm{GHz}$, $1.605 \mathrm{GHz}, 1.955 \mathrm{GHz}$, and $2.145 \mathrm{GHz}$, respectively. These modes have proved to be the natural radiating modes that can be physically excited at their own resonant frequencies.

To clearly demonstrate the effectiveness of the proposed approach, a comparison study among three different models is carried out. In the first model, only an isolated rectangular patch in free space is considered. This model has been used for CM analysis of MSA in [11] to get some physical insights of MSAs. Figure 1(a) shows the modal significances solved for this model by using the CM theory of conducting bodies. As can be seen, the patch modes exhibit very wide bandwidth, although such wide bandwidth is generally not achievable in practical MSA designs. This is mainly due to the ignorance of the ground plane in practice.

To investigate the effect of the ground plane, the second model which consists of a rectangular patch and an infinite ground plane is considered for CM analysis. The patch size is the same as that in the first model. The space between the patch and the ground plane is filled with air and the distance between them is $1.524 \mathrm{~mm}$. Figure 1 (b) shows the solved modal significance of this model. As can be seen, there are four resonant frequencies found in the frequency range of 0.8-4.0 GHz. However, they are quite different from those predicted in [2]. The discrepancy in resonant frequencies may be due to the ignorance of the dielectric material in the CM analysis.

To fully consider the true configurations of the MSA, the dielectric material $\left(\varepsilon_{r}=3.38\right)$ between the patch and the ground plane is included in the third model, and the proposed MPIE based CM formulation is implemented to solve the resonant frequencies. Figure 1(c) shows the modal significances of the third model. The CMs are sorted over the whole frequency band by using the modal tracking technique in [16]. Regarding the physical meaning of the modal significance, four resonances are observed over the frequency band. They are located at $1.08 \mathrm{GHz}, 1.60 \mathrm{GHz}$, $1.95 \mathrm{GHz}$, and $2.13 \mathrm{GHz}$, respectively. Evidently, they agree well with the resonant frequencies reported in [2].

However, rectangular MSAs with grounded dielectric substrates were previously analyzed using the CM formulation of conducting bodies $[6,11]$, where either the ground plane or the dielectric material is ignored in CM analysis. Although one can observe some radiating modes from such 


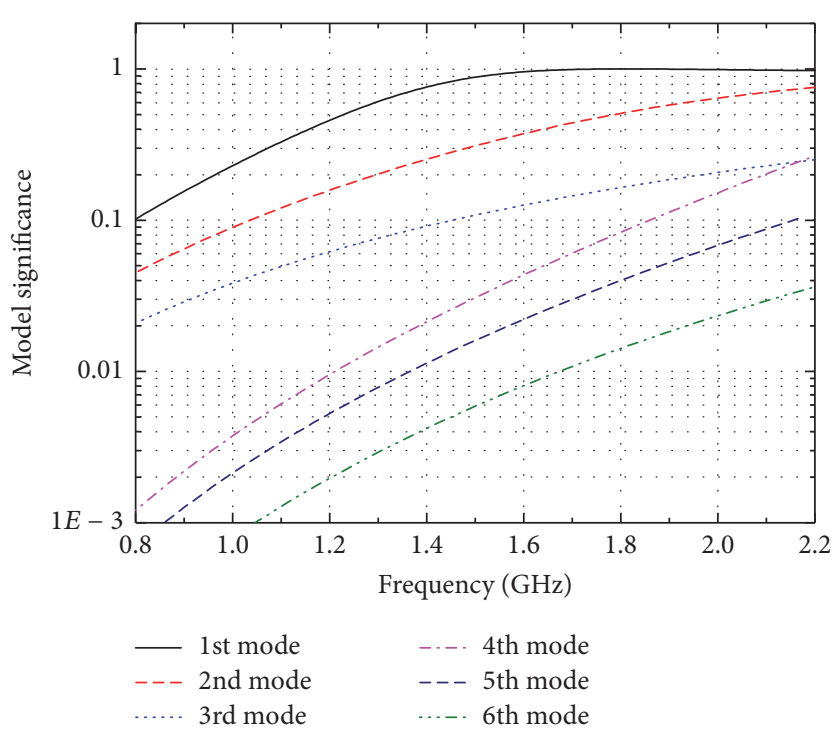

(a) Modal significances for isolated rectangular patch in free space

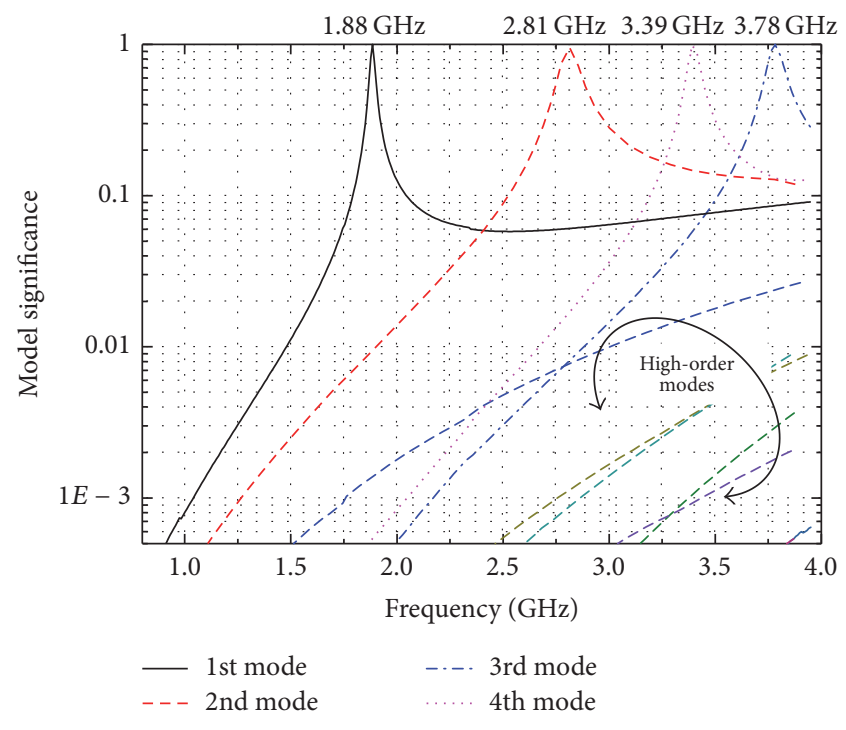

(b) Modal significances for rectangular patch with ground plane. The space between the patch and the ground plane is filled with air $\left(\varepsilon_{r}=1\right)$

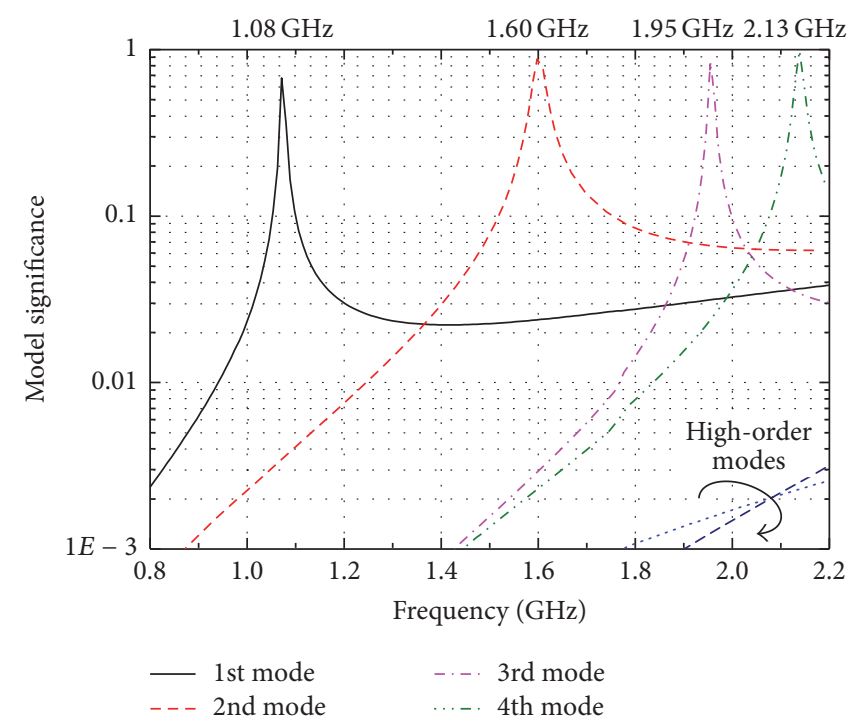

(c) Modal significances for rectangular patch with grounded dielectric substrate $\left(\varepsilon_{r}=3.38\right)$

FIGURE 1: Modal significances for the same rectangular patch but with different dielectric substrate and ground plane configurations.

$\mathrm{CM}$ analysis, there are many physical explanations that have not been fully addressed. This example clearly shows the importance of considering the grounded dielectric substrate in the CM analysis of MSAs.

Figure 2 gives the characteristic currents and fields (at their own resonant frequencies) solved from the MPIE based CM formulation for the third model. First, we observe that the four modes are orthogonal with each other, in terms of the surface current polarizations and magnitudes. Second, they agree well with the physically excited current distributions obtained by putting a coaxial probe near the corner of the patch [2]. Third, because CMs are excitation independent, which means they are inherent to geometry and material properties, hence, it is quite attractive to get these modal solutions before feeding designs. The characteristic currents in turn provide useful guidelines on how to design feedings for the excitation of each radiating mode.

For comparison purpose, the characteristic currents and fields solved from the first model (an isolated rectangular patch in free space) are plotted in Figure 3. As Figure 1(a) shows that the resonances of the isolated patch are not as evident as in Figures 1(b) and 1(c), all the CMs in Figure 3 are plotted at the center frequency $1.5 \mathrm{GHz}$. As can be seen, the modal fields of the 1st, 2nd, 4th, and 5th modes in Figure 3 are very close to the radiating modes $\mathrm{TM}_{10}, \mathrm{TM}_{01}, \mathrm{TM}_{11}$, and $\mathrm{TM}_{20}$, respectively. This is the reason that the CM theory of 


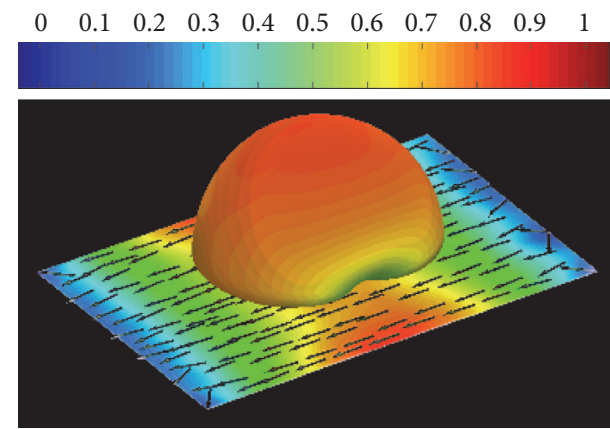

(a) $\mathrm{TM}_{10}$ (1st mode)

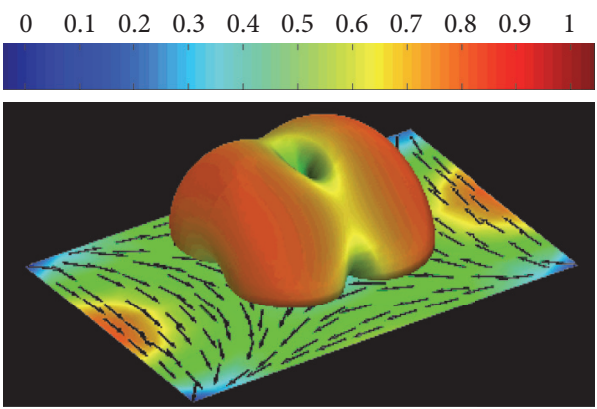

(c) $\mathrm{TM}_{11}(3 \mathrm{rd}$ mode $)$

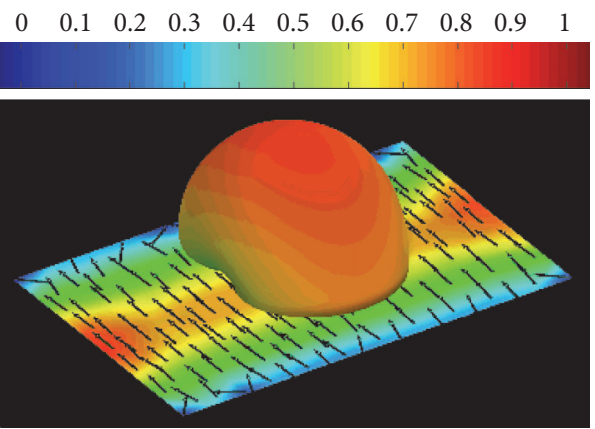

(b) $\mathrm{TM}_{01}$ (2nd mode)

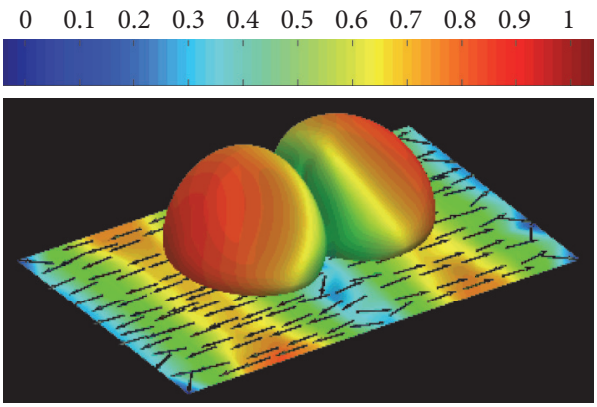

(d) $\mathrm{TM}_{20}$ (4th mode)

FIgURE 2: The characteristic currents and fields of the rectangular patch with grounded dielectric substrate $\left(\varepsilon_{r}=3.38\right)$.

conducting bodies can be used to roughly predict the modal behavior of MSAs. On the other hand, the 3rd and 6th modes do not appear in the MPIE based CM analysis results. They are defined as the inductive modes in [11] because the characteristic currents form closed loops over the two-dimensional plate and exhibit special inductive property. In [11], CabedoFabres et al. have also demonstrated that such modes did not resonate and presented an inductive contribution at each frequency [11]. However, they are not present in the case of a patch with ground plane. This comparison study clearly shows the impact of the grounded dielectric substrate and the necessity to consider the grounded dielectric substrate in CM analysis through using the MPIE formulation, especially in the case that we need to get all the true radiating modes of a MSA.

3.2. Dual-Band Circularly Polarized Antenna. Although CM theory for PEC bodies [6] has been used to investigate the circular polarization potential of an isolated triangular patch without dielectric substrate and ground, practical circularly polarized MSA with grounded substrate has not been discussed before in the framework of characteristic mode theory. The mechanism of dual-band MSA has also not been discussed through characteristic mode analysis. To fully explore the application potential of the MPIE based CM theory in more complicated microstrip antenna designs, this section focuses on the CM analysis of a dual-band circularly polarized MSA with three-layered dielectric substrates using the MPIE based CM formulation. The dual-band circularly polarized MSA was originally reported in [17], and the configuration and dimensions can be also found in the inset of Figure 4(b). A coaxial probe directly feeds the top patch through a via hole on the bottom patch. Because CMs are excitation independent, it is able to get modal solutions of MSAs without considering any specific feeding structures. Hence, the coaxial probe and the via hole are neglected in the $\mathrm{CM}$ analysis.

Figures 4(a) and 4(b) show the modal significances and characteristic angles, respectively. As can be seen, there are four resonances over the frequency band of $1.8-3.1 \mathrm{GHz}$. The first two modes coresonate in the $1.9 \mathrm{GHz}$ band, and the $3 \mathrm{rd}$ and 4 th modes coresonate in the $2.8 \mathrm{GHz}$ band. The vertical dash lines in Figures 4(a) and 4(b) indicate the frequency at which the characteristic angle differences between modes 1 and 2 and modes 3 and 4 exactly equal $90^{\circ}$. As can be seen, the first two dash lines center at about $1.9 \mathrm{GHz}$ and the 3rd and 4th dash lines center at about $2.8 \mathrm{GHz}$ band. Moreover, the modal significances of mode 1 and mode 2 between the first two dash lines are comparable with each other. The same observation is clearly seen in the $2.8 \mathrm{GHz}$ band. Considering the physical meanings of the modal significance and the characteristic angle, if the first two modes were excited simultaneously, they will provide comparable radiation power and generate far fields with a phase difference around $90^{\circ}$. Clearly, these are the radiation mechanisms of this dual-band CP antenna.

Figure 5 shows the characteristic currents and characteristic fields of the four CMs. In Figures 5(a)-5(d), the left 


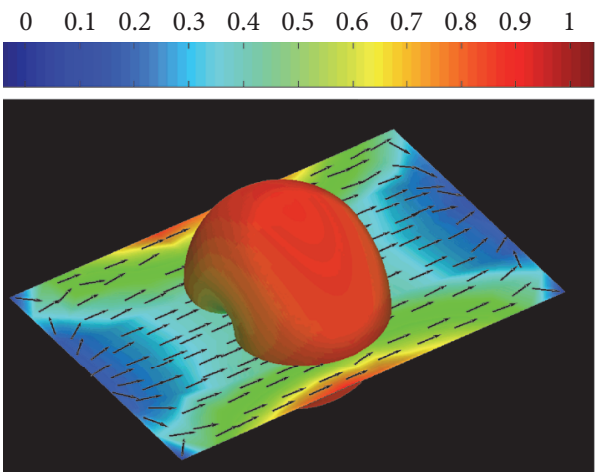

(a) 1st mode

$\begin{array}{lllllllllll}0 & 0.1 & 0.2 & 0.3 & 0.4 & 0.5 & 0.6 & 0.7 & 0.8 & 0.9 & 1\end{array}$

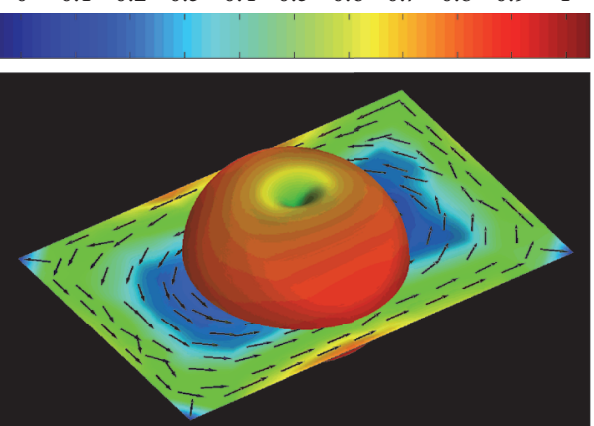

(c) 3rd mode

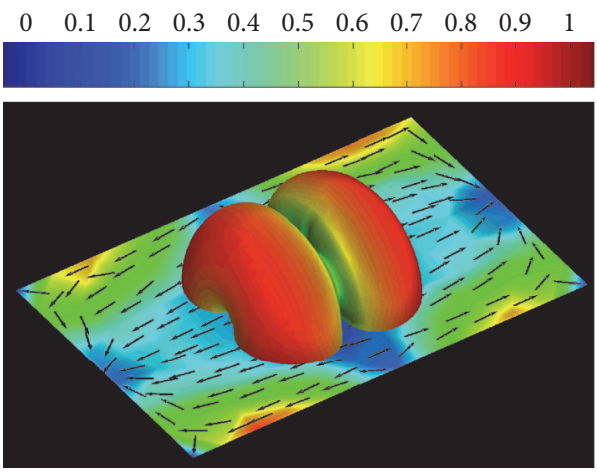

(e) 5 th mode

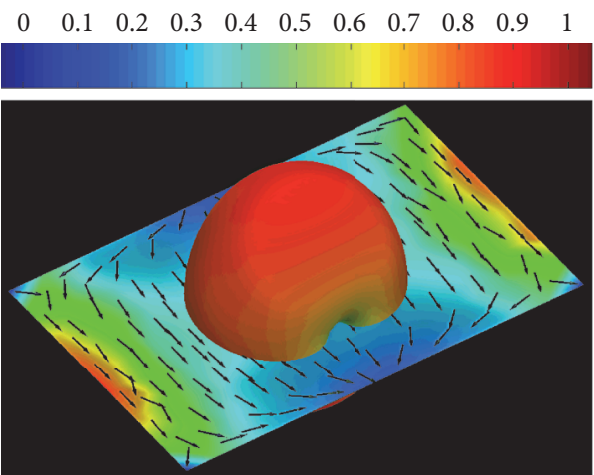

(b) 2nd mode

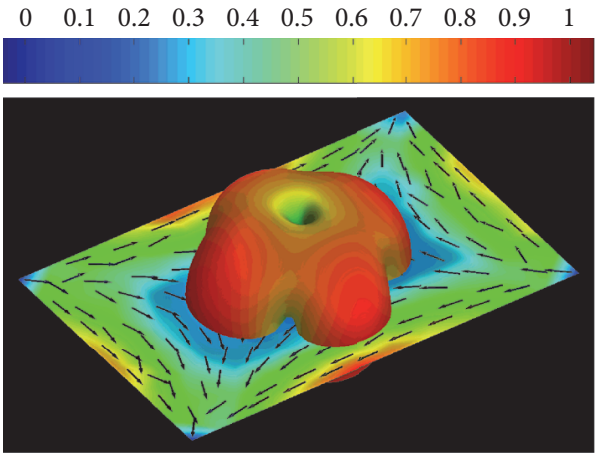

(d) 4th mode

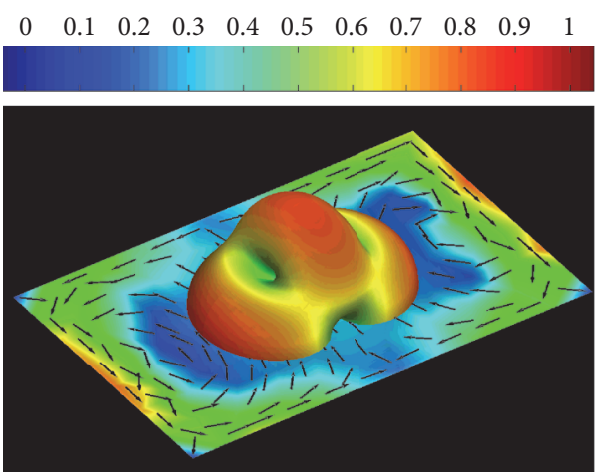

(f) 6th mode

FIgURE 3: The characteristic currents and fields of the rectangular patch in free space.

figure shows the characteristic currents on the top patch, while the right figure shows the characteristic currents on the bottom patch. As can be seen, the first two modes have orthogonal radiating currents on the bottom patch, whereas the currents on the top patch are very weak. Hence, the bottom patch dominates the radiation in the lower band. The orthogonality of the first two modes ensures the CP radiation. The characteristic currents and characteristic fields of the 3rd and 4th modes are also orthogonal with each other. The characteristic currents on the bottom and top patches have comparable current magnitudes, which indicate that both of the two patches resonate in the higher band, and contribute together to the total $\mathrm{CP}$ radiation in the $2.8 \mathrm{GHz}$ band.
In summary, the modal significance, characteristic angle, and characteristic currents clearly illustrate that $\mathrm{CP}$ radiation can be realized by properly exciting and combining coresonant CMs. Dual-band performance is also observed from the $\mathrm{CM}$ analysis results.

\section{Conclusion}

The CM theory based on the MPIE with spatial-domain GFs for multilayered media is proposed for the modal analysis of microstrip antennas with multilayered media. Against the conventional CM theory in [6], which was formulated 


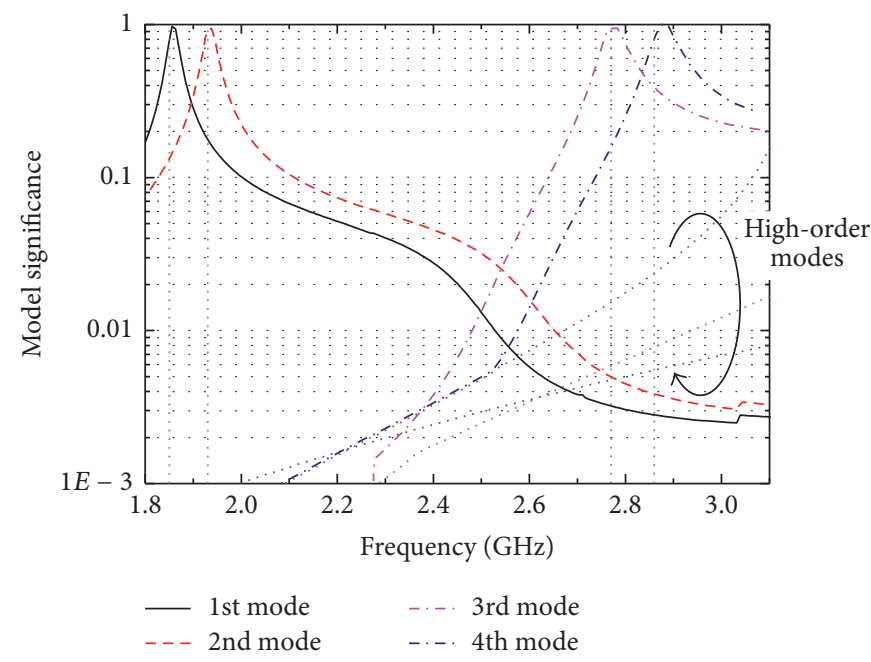

(a)

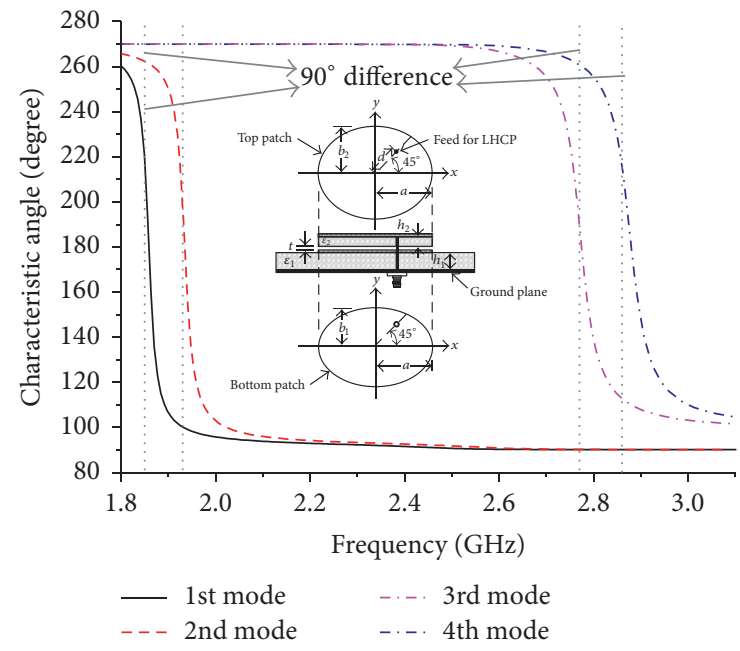

(b)

FIGURE 4: Modal significances (a) and characteristic angles (b) for the dual-band CP antenna. $\varepsilon_{1}=\varepsilon_{2}=4.4, h_{1}=1.6 \mathrm{~mm}, h_{2}=0.8 \mathrm{~mm}$, $t=0.5 \mathrm{~mm}, a=21.86 \mathrm{~mm}, b_{1} / a=0.95$, and $b_{2} / a=0.96$. The grayed dash lines indicate the frequency at which the characteristic angle differences exactly equal $90^{\circ}$.

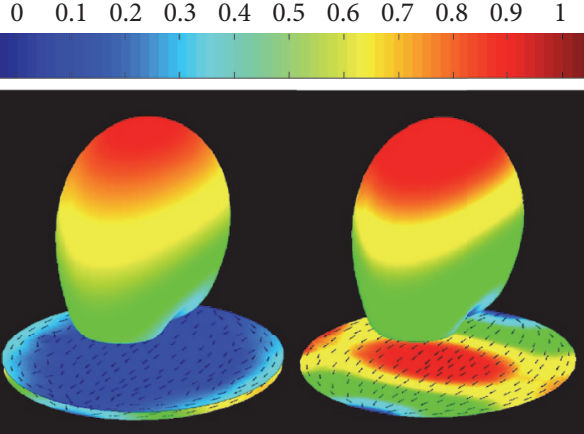

(a)
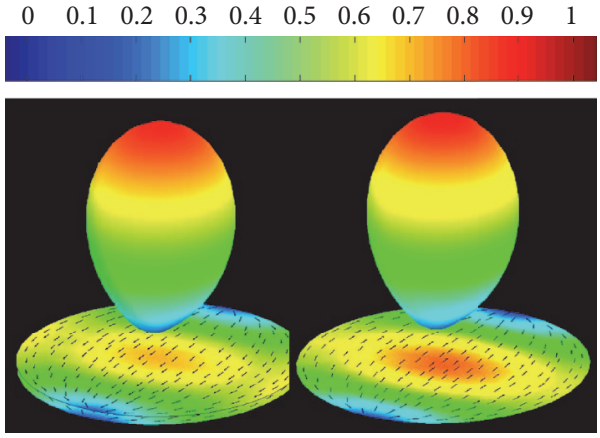

(c)

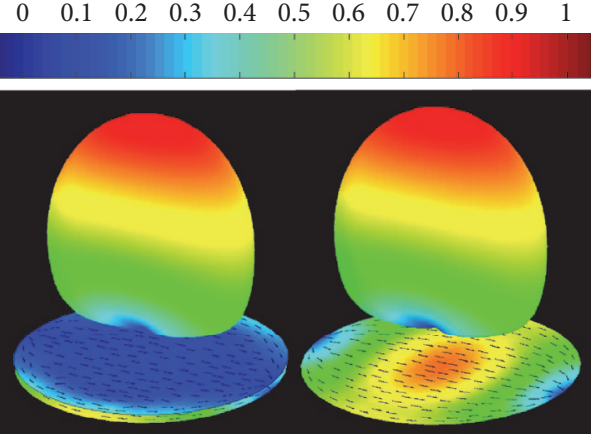

(b)

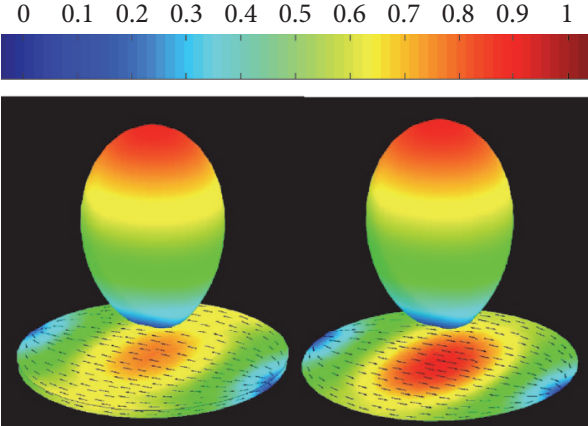

(d)

Figure 5: Characteristic currents and fields for (a) 1st mode, (b) 2nd mode, (c) 3rd mode, and (d) 4th mode. In figures (a)-(d), the left figure shows the characteristic currents on the top patch, while the right figure shows the characteristic currents on the bottom patch.

from EFIE of PEC bodies and then was borrowed for modal analysis of MSAs, the present approach fully describes the real physics of the MSA geometry. Owing to this essential difference, this method accurately solves the resonant frequencies and near/far modal fields for dominant mode as well as higher order modes. These modal analysis results are quite attractive and will systematically guide practical MSA designs. Examples are presented to show the accuracy and validity. MSAs designs with the proposed CM theory will be further addressed in our follow-up efforts. 


\section{Competing Interests}

The authors declare no conflict of interests regarding the publication of this paper.

\section{Acknowledgments}

This work is supported by the Natural Science Foundation of China under Grant no. 61671127.

\section{References}

[1] K.-F. Lee, K.-M. Luk, and J. S. Dahele, "Characteristics of the equilateral triangular patch antenna," IEEE Transactions on Antennas and Propagation, vol. 36, no. 11, pp. 1510-1518, 1988.

[2] Z. N. Chen and M. Chia, Broadband Planar Antennas, Design and Applications, John Wiley \& Sons, New York, NY, USA, 2005.

[3] M. El Yazidi, M. Himdi, and J. P. Daniel, "Transmission line analysis of nonlinear slot coupled microstrip antenna," Electronics Letters, vol. 28, no. 15, pp. 1406-1408, 1992.

[4] X. Gang, "On the resonant frequencies of microstrip antennas," IEEE Transactions on Antennas and Propagation, vol. 37, no. 2, pp. 245-247, 1989.

[5] M. D. Deshpande, D. G. Shively, and C. R. Cockrell, "Resonant frequencies of irregularly shaped microstrip antennas using method of moments," NASA Technical Paper 3386, 1993.

[6] R. Harrington and J. Mautz, "Theory of characteristic modes for conducting bodies," IEEE Transactions on Antennas and Propagation, vol. 19, no. 5, pp. 622-628, 1971.

[7] J. Eichler, P. Hazdra, M. Capek, and M. Mazanek, "Modal resonant frequencies and radiation quality factors of microstrip antennas," International Journal of Antennas and Propagation, vol. 2012, Article ID 490327, 9 pages, 2012.

[8] C. Van Niekerk and J. T. Bernhard, "Characteristic mode analysis of a shorted microstrip patch antenna," in Proceedings of the IEEE Antennas and Propagation Society International Symposium (APSURSI '12), pp. 1-2, Chicago, Ill, USA, July 2012.

[9] Y. Chen and C.-F. Wang, "Characteristic-mode-based improvement of circularly polarized U-slot and E-shaped patch antennas," IEEE Antennas and Wireless Propagation Letters, vol. 11, pp. 1474-1477, 2012.

[10] R. Garbacz and R. Turpin, "A generalized expansion for radiated and scattered fields," IEEE Transactions on Antennas and Propagation, vol. 19, no. 3, pp. 348-358, 1971.

[11] M. Cabedo-Fabres, E. Antonino-Daviu, A. Valero-Nogueira, and M. F. Bataller, "The theory of characteristic modes revisited: a contribution to the design of antennas for modern applications," IEEE Antennas and Propagation Magazine, vol. 49, no. 5, pp. 52-68, 2007.

[12] Y. Chen and C.-F. Wang, "HF band shipboard antenna design using characteristic modes," Institute of Electrical and Electronics Engineers. Transactions on Antennas and Propagation, vol. 63, no. 3, pp. 1004-1013, 2015.

[13] Y. Chen and C.-F. Wang, "Electrically small UAV antenna design using characteristic modes," IEEE Transactions on Antennas and Propagation, vol. 62, no. 2, pp. 535-545, 2014.

[14] D. G. Fang, J. J. Yang, and G. Y. Delisle, "Discrete image theory for horizontal electric dipoles in a multilayered medium," IEE Proceedings H: Microwaves, Antennas and Propagation, vol. 135, no. 5, pp. 297-303, 1988.
[15] F. Ling, C.-F. Wang, and J.-M. Jin, "An efficient algorithm for analyzing large-scale microstrip structures using adaptive integral method combined with discrete complex-image method," IEEE Transactions on Microwave Theory and Techniques, vol. 48, no. 5, pp. 832-839, 2000.

[16] B. D. Raines and R. G. Rojas, "Wideband characteristic mode tracking," IEEE Transactions on Antennas and Propagation, vol. 60, no. 7, pp. 3537-3541, 2012.

[17] J.-Y. Jan and K.-L. Wong, "Dual-band circularly polarized stacked elliptic microstrip antenna," Microwave and Optical Technology Letters, vol. 24, no. 5, pp. 354-357, 2000. 


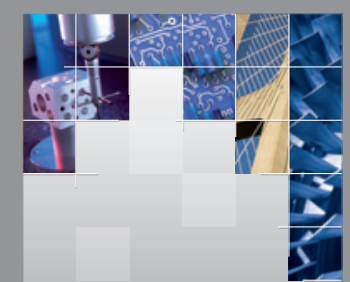

\section{Enfincering}
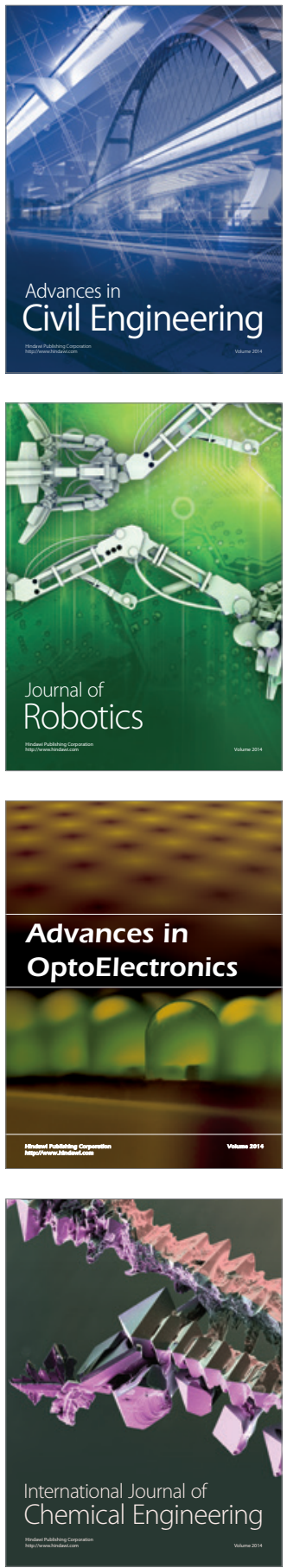

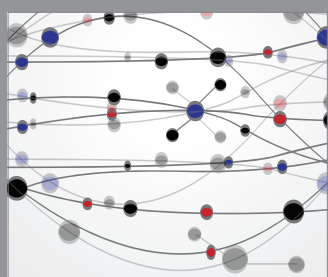

The Scientific World Journal

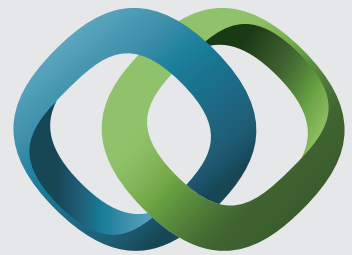

\section{Hindawi}

Submit your manuscripts at

http://www.hindawi.com
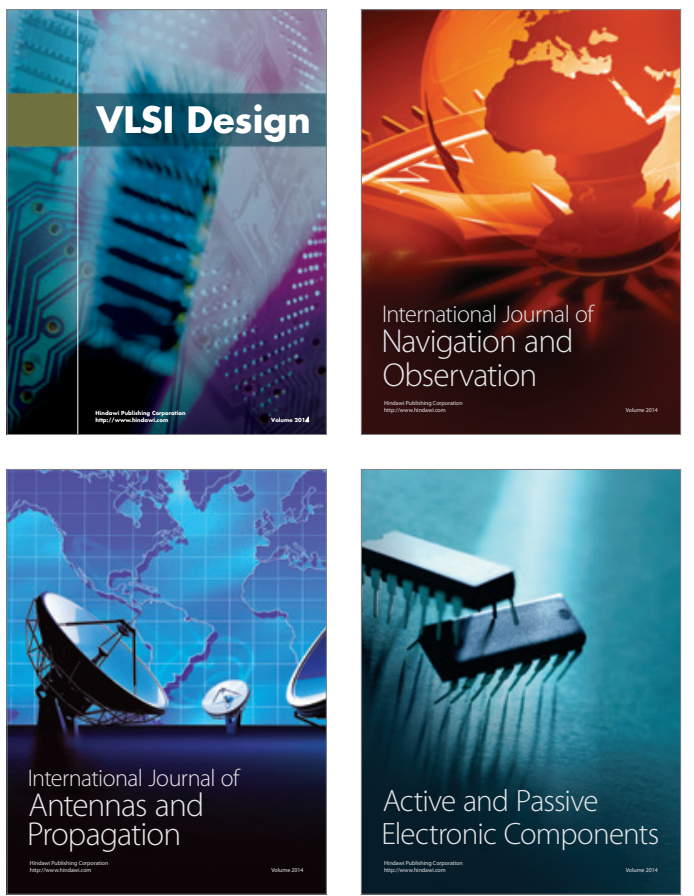
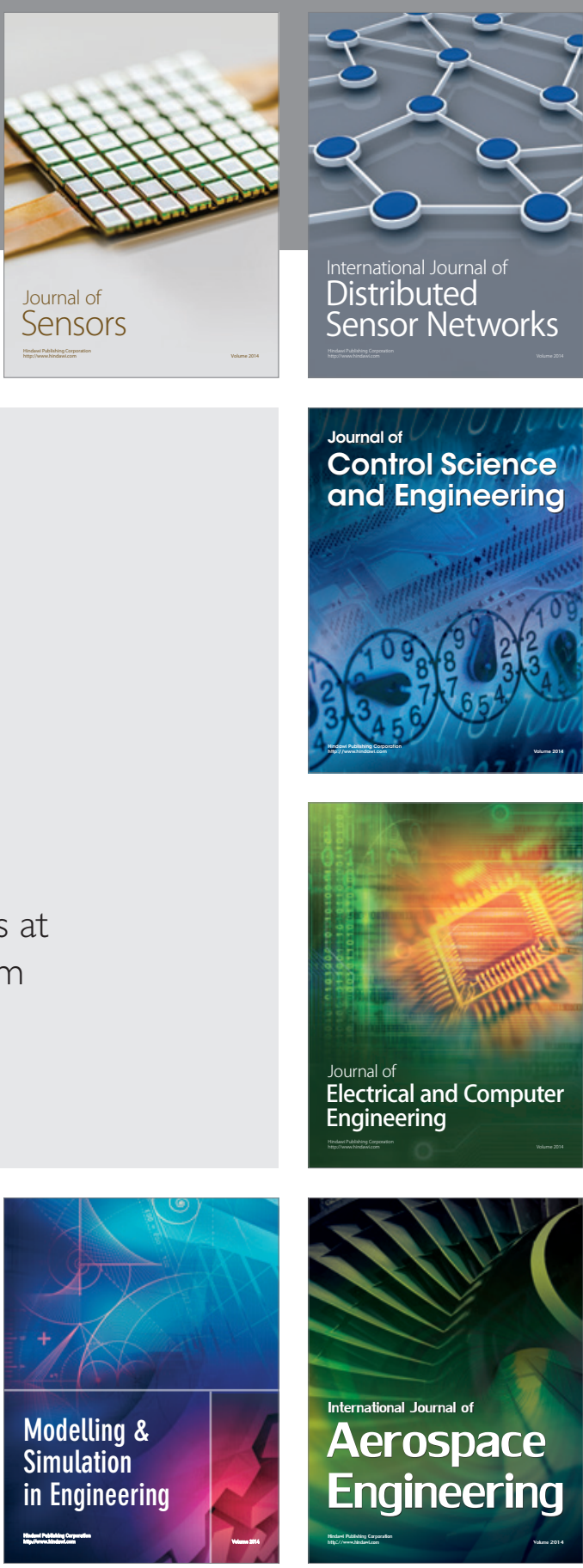

International Journal of

Distributed

Sensor Networks

Journal of

Control Science

and Engineering
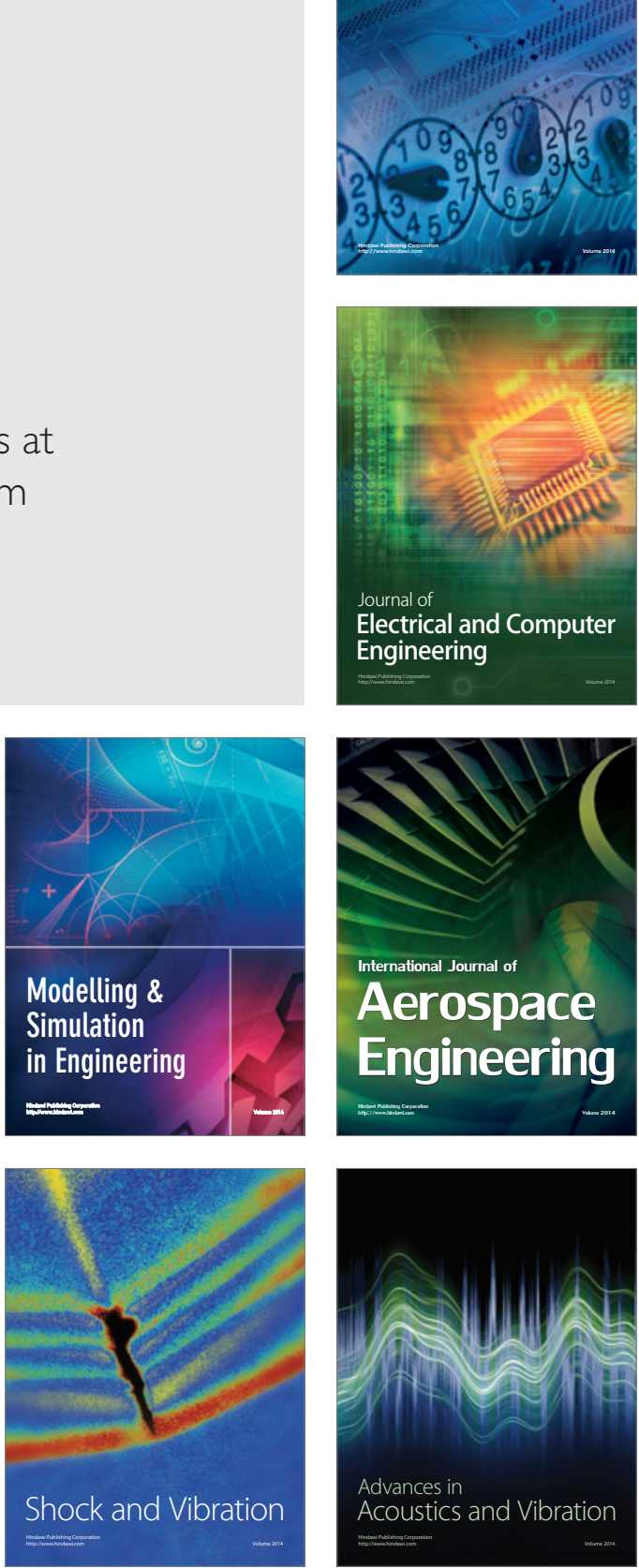